\title{
THE CONNES SPECTRUM OF GROUP ACTIONS AND GROUP GRADINGS FOR CERTAIN QUOTIENT RINGS
}

\author{
JAMES OSTERBURG AND XUE YAO
}

\begin{abstract}
Let $H$ be a finite-dimensional, semisimple Hopf algebra over an algebraically closed field $K$ where $H$ is either commutative or cocommutative. We let $A$ be an $H$-module algebra which is semiprime right Goldie. We show that the Connes spectrum of $H$ acting on $A$ is the Connes spectrum of $H$ acting on the classical quotient ring of $A$. In our last section, we define a symmetric quotient ring and show that the Connes spectrum of the ring and its quotient ring are the same. Finally, we apply our results to finite group actions and group gradings.
\end{abstract}

\section{INTRODUCTION}

This paper basically concerns finite group actions and group gradings, and we use a Hopf algebra setting to provide a unified proof of both situations. We begin by discussing some special cases of our results. Let $K$ be an algebraically closed field; and assume $A$ is a semiprime, Goldie $K$-algebra with quotient ring $Q$. Let $G$ be a finite group that acts as $K$-automorphisms on $A$, where the order of $G$ is nonzero in $K$, or let $G$ grade $A$. Then $G$ acts as $K$ automorphims on $Q$ or $G$ grades $Q$. It is clear that the action or the grading of $G$ on $A$ is very similar to the action or the grading of $G$ on $Q$. These situations have been studied in several papers.

From [OPQ], the Connes spectrum of a group action consists of certain irreducible representations of $G$ and the Connes spectrum of a group grading consists of certain group elements of $G$. In broad terms, the Connes spectrum of a group action or a group grading is a measure of the amount of the action or the amount of the grading. A major purpose of this paper is to prove that the Connes spectrum of $G$ acting on $A$ is the Connes spectrum of $G$ acting on $Q$ and to show the Connes spectrum of a $G$ grading $A$ is the Connes spectrum of $G$ grading $Q$. This precisely quantifies the feeling that the action or the grading of $G$ on $A$ is very similar to the action or the grading of $G$ on $Q$.

Received by the editors October 25, 1993 and, in revised form, April 25, 1994; originally communicated to the Proceedings of the AMS by Ken Goodearl.

1991 Mathematics Subject Classification. Primary 16W30; Secondary 16W20.

Key words and phrases. Connes spectrum, Hopf algebra actions, Goldie rings, symmetric quotient ring.

The authors thank S. Montgomery for her useful comments.

The first author was partially supported by NSA grant MDA 904-92-H-3080. The second author was supported by a Taft fellowship from the University of Cincinnati. 
We are able to extend our results to cocommutative or commutative Hopf algebra actions. In fact, a consequence of Theorems 2.5 and 2.8 is

Theorem A. Assume $H$ is a finite-dimensional, semisimple Hopf algebra over an algebraically closed field $K$. Let $H$ be either commutative or cocommutative. Let $A$ be a semiprime $H$-module algebra with identity. Assume $A$ is a semiprime right Goldie ring with quotient ring $Q$. Then the Connes spectrum of $H$ acting on $A$ is the Connes spectrum of $H$ acting on $Q$. Furthermore, the smash product $A \# H$ is prime if and only if $Q \# H$ is simple.

There are advantages to working in the classical quotient ring. For example, the hereditary subalgebras of $Q$ are of the form $e Q e$, where $e^{2}=e$ and $e$ is fixed under the action of $H$. In the group action case, the Connes spectrum is full precisely when the action of $G$ is Galois on each hereditary subalgebra. This is called an hereditarily saturated action in [Ph]. We extend this notion to Hopf algebra actions in Theorem 2.9.

We let $A$ be a commutative domain with a finite group $G$ acting on $A$. We let $W$ be the subgroup of $G$ that acts trivially on $A$. We show that the Connes spectrum of $G$ acting on $A$ is the irreducible representation of $G / W$. Thus the Connes spectrum consists of the trivial representation if and only if the action of $G$ on $A$ is trivial and the spectrum is full precisely when the action is faithful. This result is not true for matrix rings over $A$, see [OP2].

For Goldie rings, a major difficulty in the general Hopf case is extending the action of $H$ to the quotient ring. In the second part of this paper, we study a Martindale type of quotient ring where the action can always be extended. By [MS, Theorem 3.9] the action can be extended to this quotient ring when the antipode is bijective. Thus for commutative or cocommutative Hopf algebras, the action on $A$ can be extended to the $H$-symmetric quotient ring of $\mathrm{A}, Q_{H}$. See also [C, M2]. A consequence of Corollary 3.4 is

Theorem B. Let $H$ be a finite-dimensional, semisimple commutative or cocommutative Hopf algebra over an algebraically closed field $K$. Let $A$ be an $H$ module algebra, and assume $A$ is an $H$-semiprime ring. Then $A \# H$ is prime if and only if $Q_{H} \# H$ is prime.

We conclude by applying our results to finite-dimensional pointed cocommutative Hopf algebra actions. In this case, $Q_{H}(A)=Q(A)$, where $Q(A)$ is the symmetric quotient ring. See [MS, Example 3.5]. As is well known, cocommutative Hopf algebras over algebraically closed fields are pointed, see [MS, §5.6]. So the next result follows easily from Theorem B.

Theorem C. Let $H$ be a finite-dimensional, semisimple cocommutative Hopf algebra over an algebraically closed field $K$. Let $A$ be an $H$-module algebra, and assume $A$ is an $H$-semiprime ring. Then $A \# H$ is prime if and only if $Q \# H$ is prime.

Because group algebras of finite groups are pointed cocommutative Hopf algebras, the above result basically extends a result of D. S. Passman [P2, Theorem 15.9]. See also Corollary 3.5 below.

\section{Hopf algebra aCtions ON Goldie RINGS}

We will briefly introduce the Connes spectrum for a Hopf algebra action, but the reader is referred to [OPQ] for more information. We let $H$ be a finite- 
dimensional Hopf $K$-algebra, where $K$ is a splitting field of $H$. We assume $H$ is strongly semiprime, as in [OPQ]. In particular, strongly semiprime algebras are semisimple. Let $A$ be an $H$-module $K$-algebra with identity. We denote the action of $h \in H$ on $a \in A$ by $h \cdot a$. Let $J$ be a nonzero ideal of the fixed algebra $A^{H}$, where

$$
A^{H}=\{a \in A \mid h \cdot a=\varepsilon(h) a \text { for all } h \in H\} .
$$

Any subalgebra of the form $J A J$ is called a hereditary subalgebra of $A$. By [OPQ, Lemma 4.5], if $A$ is $H$-semiprime and $B$ is of this form, then $B$ reg $B$, which means that the left and right annihilators of $B$ in $B$ are zero. Note that $H$ acts on any matrix algebra over $B$ by acting on the coordinates, i.e. $h \cdot\left(b_{i, j}\right)=\left(h \cdot b_{i, j}\right)$.

Let $\pi: H \rightarrow M_{d_{\pi}}(K)$ be an irreducible representation of $H$. We denote the set of irreducible representations of $H$ by $\operatorname{Irr}(H)$. Let $S$ be the antipode of $H$ and $\varepsilon$ denote the counit. We use the summation notation. The following definitions are the central ones.

$$
\begin{aligned}
& B_{\pi}^{m}=\left\{X \in M_{d_{\pi}}(B) \mid \varepsilon(h) X=\sum_{(h)} \pi\left(h_{3}\right)\left(h_{1} \cdot X\right) \pi\left(S^{-1}\left(h_{2}\right)\right)\right\}, \\
& B_{\pi}^{l}=\left\{X \in M_{d_{\pi}}(B) \mid \varepsilon(h) X=\sum_{(h)} \pi\left(h_{2}\right)\left(h_{1} \cdot X\right)\right\}, \\
& B_{\pi}^{r}=\left\{X \in M_{d_{\pi}}(B) \mid \varepsilon(h) X=\sum_{(h)}\left(h_{1} \cdot X\right) \pi\left(S^{-1}\left(h_{2}\right)\right)\right\},
\end{aligned}
$$

where the right-hand formulas hold for all $h \in H$. As is shown in [OPQ], $B_{\pi}^{m}$ is a ring and $B_{\pi}^{l} B_{\pi}^{r}$ is a two-sided ideal of this ring. We define the Connes spectrum of $H$ on $A$ to be

$$
\operatorname{CS}(A, H)=\left\{\pi \in \operatorname{Irr}(H) \mid B_{\pi}^{l} B_{\pi}^{r} \text { reg } B_{\pi}^{m} \text { for all } B\right\} .
$$

Paper [OPQ, Theorem 1.6] goes on to prove that the smash product $A \# H$ is prime precisely when $A$ is $H$-prime and $\operatorname{CS}(A, H)=\operatorname{Irr}(H)$.

We let $e$ denote the principal idempotent of $H$. In other words, $\mathrm{Ke}$ is the complement of the kernel of the counit. We let $\operatorname{Tr}(a)=e \cdot a$ and note $\operatorname{Tr}: A \rightarrow A^{H}$. If $A \# H$ is semiprime, then $\operatorname{Tr}(R) \neq 0$ for any nonzero $H$-stable right ideal $R$ of $A$. See [OPQ, Propostion 4.3].

Our next result is similar to unpublished results of D. Fischman. Our proof is modeled on [P2, Theorem 26.9]. Let us note that if $A$ is semiprime and $H$ is strongly semiprime, then $A \# H$ is semiprime, by definition.

Theorem 2.1. Assume $H$ is a finite-dimensional, strongly semiprime Hopf algebra over a splitting field $K$. Let $A$ be a semiprime $H$-module algebra with identity. If $A$ is a right Goldie ring, then $A^{H}$ is a right Goldie ring. Furthermore, if every essential right ideal of $A$ contains an essential $H$-stable right ideal of $A$, then the classical quotient ring of $A, Q(A)$, is $A T^{-1}$, where $T$ is the set of regular elements in $A^{H}$ and $Q\left(A^{H}\right)=Q(A)^{H}$.

Proof. We assume $A$ is Goldie. Since $A^{H} \subseteq A \subseteq Q(A)$ and $Q(A)$ satisfies the maximum condition, we can conclude that $A^{H}$ satisfies the maximum condition on right annihilators of $A$. Let $R_{1} \dot{+} \cdots+R_{n}$ be a direct sum of right ideals of $A^{H}$. Then $R_{i} A$ for $1 \leq i \leq n$ is certainly an $H$-stable right ideal of $A$. 
Now

$$
\operatorname{Tr}\left(R_{i} A \cap \sum_{j \neq i} R_{j} A\right) \subseteq\left(R_{i} \cap \sum_{j \neq i} R_{j}\right)=0
$$

Thus $R_{i} A \cap \sum_{j \neq i} R_{j} A=0$ by [OPQ, Lemma 4.3]. We have shown that $A^{H}$ is right Goldie.

To show $Q(A)=A T^{-1}$ we verify the conditions of [P, Lemma 26.8] hold. To this end, let $t \in T$. We show $t A$ ess $A$ or $t A$ is essential in $A$. Since $A$ is Goldie, this is equivalent to showing $t$ is regular in $A$ [GW, Proposition 5.9]. Let $L$ be the left annihilator of $t$ in $A$. Then $L$ is an $H$-stable ideal with $\operatorname{Tr}(L)=0$ and so $L=0$. Similarly the right annihilator of $t$ is zero. Thus $t$ is regular in $A$.

Let $E$ be an essential right ideal of $A$ with $\bar{E}$ an essential $H$-stable right ideal of $A$ contained in $E$. We will show $E \cap T \neq \varnothing$. This will follow if we show $E \cap A^{H}$ ess $A^{H}$, since $A^{H}$ is Goldie and $\bar{E} \cap A^{H} \subseteq E \cap A^{H}$. Let $0 \neq W$ be a right ideal of $A^{H}$, then $W A \cap \bar{E} \neq 0$. So $0 \neq \operatorname{Tr}(W A \cap \bar{E}) \subseteq$ $W A^{H} \cap \operatorname{Tr}(\bar{E}) \subseteq W \cap\left(\bar{E} \cap A^{H}\right)$. Thus $E \cap A^{H}$ ess $A^{H}$, so $E \cap T \neq \varnothing$. Thus the conditions of [P2, Lemma 26.8] hold .

Extending the action of $H$ to $Q(A)$. We extend the action of $H$ from $A$ to $A T^{-1}$ as follows:

$$
h \cdot\left(a t^{-1}\right)=(h \cdot a) t^{-1}
$$

for $a \in A, t \in T$, and $h \in H$. In fact, this is the only way to extend the action. To see this, let $\square$ be an action of $H$ on $A T^{-1}$ such that $h \boxminus a=h \cdot a$ for $a \in A$ and $h \in H$. Let $t \in T$, then $\varepsilon(h) 1=h \cdot 1=h \boxminus\left(t t^{-1}\right)=\sum_{(h)}\left(h_{1} \oslash t\right)\left(h_{2} \boxminus t^{-1}\right)=$ $\sum_{(h)} \varepsilon\left(h_{1}\right) t\left(h_{2} \boxminus t^{-1}\right)=t\left(h \boxminus t^{-1}\right)$. Thus we have shown $h \boxminus t^{-1}=\varepsilon(h) t^{-1}$. Hence for $a \in A, h \boxminus\left(a t^{-1}\right)=\sum_{(h)}(h \cdot a)\left(t^{-1}\right)$.

When $Q(A)=A T^{-1}$. Let $H$ be as in Theorem 1, and assume $A$ is semiprime Goldie and an $H$-module algebra. Then if the classical quotient ring of $A$ is $A T^{-1}$, every essential right ideal $E$ of $A$ contains an $H$-stable essential ideal $E$. To see this, note $E$ contains a regular element $s \in A$. So $s^{-1}=a t^{-1}$ for some $a \in A, t \in T$. Thus $t A \subseteq s A \subseteq E$ and $t A$ is $H$-stable since $t \in A^{H}$.

The next two lemmas concern the passage between hereditary subalgebras of $A$ and $Q(A)$ and the components of the Connes spectrum.

Lemma 2.2. Let $A$ be a right Goldie ring with quotient ring $Q(A)$, and assume the other hypotheses of Theorem 1 hold. Then:

(1) If $J$ is a nonzero ideal of $A^{H}$, then $\hat{J}=J T^{-1}$ is a nonzero ideal of $Q(A)^{H}$.

(2) Let $A$ be semiprime, Goldie with quotient ring $Q(A)$. Let $0 \neq \hat{J}$ be an ideal of $Q(A)^{H}$. Then $J=\hat{J} \cap A$ is a nonzero ideal of $A^{H}$.

(3) If $B=J A J$ and $\hat{B}=\hat{J} Q(A) \hat{J}$, then $B \subseteq \hat{B}$.

(4) Let $s, t \in T$; then $t\left(\hat{B}_{\pi}^{l}\right) s \subseteq \hat{B}_{\pi}^{l}$, and $t\left(B_{\pi}^{l}\right) s \subseteq B_{\pi}^{l}, \quad t\left(\hat{B}_{\pi}^{r}\right) s \subseteq \hat{B}_{\pi}^{r}$ and $t\left(B_{\pi}^{r}\right) s \subseteq B_{\pi}^{r}$ and $t\left(\hat{B}_{\pi}^{m}\right) s \subseteq \hat{B}_{\pi}^{m}$ and $t\left(B_{\pi}^{m}\right) s \subseteq B_{\pi}^{m}$.

Proof. (1) See [GW, Theorem 9.17(b)].

(2) See [GW, Theorem 9.17(a)].

(3) This follows because $J \subset \hat{J}$. 
(4) These follow because $J$ and $\hat{J}$ are ideals and the definitions of $B_{\pi}^{l}$, $B_{\pi}^{r}$, and $B_{\pi}^{m}$.

Lemma 2.3. Assume $H$ is a finite-dimensional, strongly semiprime Hopf algebra over a splitting field $K$. Let $A$ be an $H$-semiprime $H$-module algebra with identity. Let $\pi: H \rightarrow M_{d_{\pi}}(K)$ be an irreducible representation of $H$.

(1) Let $J$ be a nonzero ideal of $A^{H}$, and let $B=J A J$. Then $B_{\pi}^{m}$ is nonzero and semiprime. Thus the left and right annihilators of $B_{\pi}^{l} B_{\pi}^{r}$ in $B_{\pi}^{m}$ are equal.

(1) Assume $A$ is right Goldie with quotient ring $Q$. Let $\hat{J}$ be a nonzero ideal of $Q^{H}$, and let $\hat{B}=\hat{J} Q \hat{J}$. Then $\hat{B}_{\pi}^{m}$ is nonzero and semiprime. Thus the left and right annihilators of $\hat{B}_{\pi}^{l} \hat{B}_{\pi}^{r}$ in $\hat{B}_{\pi}^{m}$ are equal.

Proof. We will just prove (1), because the proof of (2) is similar. To begin, $J^{2}$ when viewed as diagonal matrices is contained in $B_{\pi}^{m}$ and thus $B_{\pi}^{m} \neq 0$. Now $B$ is semiprime and $B \# H$ is semiprime by [OPQ, Proposition 4.3 and Lemma 4.4]. Thus $(B \# H)^{H}$ is semiprime by [OPQ, Proposition 4.3], but $(B \# H)^{H} \simeq$ $\oplus \sum_{\pi \in \operatorname{Irr}(H)} B_{\pi}^{m}$ by [OPQ, Lemma 2.5 and Lemma 5.2]. Since a direct summand of a semiprime ring is semiprime, the proof is finished.

The consequences of the next result are some of the main results of this paper.

Theorem 2.4. Assume $H$ is a finite-dimensional, strongly semiprime Hopf algebra over a splitting field $K$. Let $A$ be a semiprime $H$-module algebra with identity, such that $A$ is a right Goldie ring. Assume that every essential right ideal of $A$ contains an $H$-stable essential right ideal. Then $\operatorname{CS}(A, H)=\operatorname{CS}(A, H)=$ $\operatorname{CS}(Q(A), H)$, where $Q(A)$ is the classical quotient ring of $A$.

Proof. Let $Q=Q(A)$. Assume $\pi \notin \operatorname{CS}(Q(A), H)$; so by Lemma 3 there is a $\hat{B}=\hat{J} Q \hat{J}$ with $\hat{J} \neq 0$ an ideal of $Q^{H}$, such that $\operatorname{ann}_{\hat{B}_{\pi}^{m}} \hat{B}_{\pi}^{l} \hat{B}_{\pi}^{r} \neq 0$. Denote this annihilator by $X$, and let $0 \neq z \in X$.

Since $Q^{H}$ is semisimple, there is a central idempotent $f \in Q^{H}$ such that $\hat{J}=Q^{H} f$. We let $J=\hat{J} \cap A$ and note there is a $t \in T$ such that $0 \neq f t=$ $t f \in J$. Thus by Lemma 2 and the regularity of $t$, we have that $0 \neq t z \in \hat{B}_{\pi}^{m}$ and note that $t z$ is a matrix with entries from $J Q f$. Say $t z=\left(q_{i, j} f\right)$ with $q_{i, j} f=\sum_{k} j_{k} q_{k}^{*}$, where $j_{k} \in J, \quad q_{k}^{*} \in Q$. The entries $q_{k}^{*}$ have a common denominator $s \in T$, by [GW, Lemma 9.2(b)]. Thus $q_{i, j} f s \in J A$.

Now we consider tzst and note it is nonzero because st is regular. But

$$
t z s t=\left(\left[q_{i, j} f^{2}\right] s t\right)=\left(\left[q_{i, j} f s\right][f t]\right) .
$$

Note we have used that $f$ commutes with $s, t \in T \subseteq Q^{H}$. Since $q_{i, j} f s \in J A$ and $f t \in J$, we see that $0 \neq t z s t \in B_{\pi}^{m}$.

Now by Lemma $2, B_{\pi}^{l} B_{\pi}^{r} \subseteq \hat{B}_{\pi}^{l} \hat{B}_{\pi}^{r}$ and so

$$
\text { tzst } B_{\pi}^{l} B_{\pi}^{r} \subseteq t z \hat{B}_{\pi}^{l} \hat{B}_{\pi}^{r}=0 .
$$

Thus $\pi \notin \operatorname{CS}(A, H)$.

Conversely, we assume $\pi \notin \operatorname{CS}(A, H)$. So by Lemma 3 , there exists $0 \neq J$ an ideal of $A^{H}$ such that if $B=J A J$, then $Z=\operatorname{ann}_{B_{\pi}^{m}} B_{\pi}^{l} B_{\pi}^{r} \neq 0$. Let $0 \neq z \in Z$. 
Let $\hat{J}=J T^{-1}$ and $\hat{B}=\hat{J} Q(A) \hat{J}$. Then by Lemma $2, \hat{B}$ is a hereditary subalgebra of $Q(A)$. Let $x \in \hat{B}_{\pi}^{l}$ and $y \in \hat{B}_{\pi}^{r}$. There is an $s \in T$ such that $x s \in B_{\pi}^{l}$ and $s^{-1} y \in \hat{B}_{\pi}^{r}$, by Lemma 2. Now find $t \in T$ such that $s^{-1} y t \in B_{\pi}^{r}$. Then

$$
x y t=[x s]\left[s^{-1} y t\right] \in B_{\pi}^{l} B_{\pi}^{r} .
$$

Since $z$ annihilates this from the left and $t$ is regular, we have $z x y=0$. Thus $\operatorname{ann}_{\hat{B}_{n}^{m}} \hat{B}_{\pi}^{l} \hat{B}_{\pi}^{r} \neq 0$ and hence $\pi \notin \operatorname{CS}(Q(A), H)$.

The above result will provide a single proof of the equality of the Connes spectra for commutative and cocommutative Hopf algebra actions after we verify the hypotheses of Theorem 2.4 for these actions. We do this in the following result, and we use the structure theory of semisimple, commutative, or cocommutative Hopf algebras.

Theorem 2.5. Let $H$ be a finite-dimensional, semisimple Hopf algebra over a splitting field $K$. We assume $H$ is either commutative or cocommutative. In the cocommutative situation, we assume $K$ is a perfect field. Let $A$ be an $\mathrm{H}$ module algebra with an identity that is a semiprime, right Goldie ring. Then $\operatorname{CS}(A, H)=\operatorname{CS}(Q(A), H)$, where $Q(A)$ is the classical quotient ring of $A$.

Proof. In both cases, we must show two things. The first is that commutative and cocommutative Hopf algebras are strongly semiprime and that an essential right ideal of $A, E$ contains an $H$-stable, essential right ideal $\bar{E}$. We denote $Q(A)$ by $Q$.

Step 1 (the graded case). Let $H=K G^{*}$. Because $E$ is essential, there is a regular element $s \in E$ [GW, Proposition 5.9]. But $Q=A T^{-1}$ where $T$ consists of the regular elements of $A_{1}$ [CR, Theorem 1.7]. So $s^{-1}=a t^{-1}$ for $t \in T, a \in A$. Thus $t A \subseteq s a A \subseteq E$ and $t A$ is essential because $t$ is regular in $A$ [CR, Corollary 1.18]. Since $t \in A_{1}$, we have $t A$ is $H=K G^{*}$-stable.

Step 2 ( $H$ is commutative). By [M3, Theorem 2.3.1] $H=K G^{*}$. Thus Step 1 shows essential right ideals contain $H$-stable essential right ideals. Also $H$ is strongly semiprime by [P2, Theorem 4.8].

Step $3\left(H=K P^{*} \# K G\right)$. The characteristic of $K$ is $p \geq 0, \quad P$ is a finite $p$-group, and $U=K P^{*}$. So $U$ is the dual of a group algebra. Also $G$ is a finite group with $p \nmid|G|$ and $V=K G$. So $V$ is a group algebra and $H=U \# V$. We will show the condition on essential ideals holds by using the following ideas from [C]. Let $I$ be a right ideal of $A$, and define

$$
(I: H)=\{a \in A \mid h \cdot a \in I \text { for all } h \in H\} .
$$

It is easily checked that $(I: H)$ is a $H$-stable right ideal of A and $(I: H) \subseteq I$, since $a=1 \cdot a \in I$. It is also easily checked that $((I: U): V)=(I: H)$.

Let $E$ be an essential right ideal of $A$. Then by Step $1, E^{\prime} \subseteq E$ where $E^{\prime}$ is an $U$-stable essential right ideal. But $E^{\prime} \subseteq(E: U)$. So $(E: \bar{U})$ is $U$-stable and essential as a right ideal.

Now $E^{\prime \prime}=\bigcap_{g \in G}[(E: U)]^{g}$ is essential, since $G$ is a finite group. We note $E^{\prime \prime} \subseteq((E: U): V)$ since $E^{\prime \prime}$ is $V$-stable. Thus $N=((E: U): V)$ is essential. Since $N=(E: H)$, we have shown that essential right ideals of $A$ contain essential $H$-stable right ideals. 
Step 4 ( $K$ perfect, $L$ a finite field extension of $K$ ). We know that $L$ is separable. Thus $\tilde{H}=H \otimes_{K} L$ is semisimple [P1, Theorem 7.3.9] and $L$ splits $\tilde{H}$.

Let $\tilde{A}=A \otimes_{K} L$, which we show is semiprime, Goldie by proving $\tilde{A}$ is a right order in $\tilde{Q}=Q \otimes_{K} L$ [GW, p. 87]. We begin by noting $\tilde{Q}$ is semisimple, since $L$ is perfect. We let $x \in \tilde{A}$ be regular and assume $x$ is a zero divisor in $\tilde{Q}$. Say $x y=0$ for $0 \neq y \in \tilde{Q}$. Then by [GW, Lemma 5.1] there is $b$ regular in $A$ such that $0 \neq z=y(b \otimes 1) \in \tilde{A}$. Thus $x$ is not regular in $\tilde{A}$. This contradiction shows $x$ is regular in $\tilde{Q}$, but regular elements are invertible in semisimple rings.

Next we must show that if $\tilde{q} \in \tilde{Q}$, then $\tilde{q}=\tilde{a} \tilde{b}^{-1}$ for $\tilde{a} \in \tilde{A}$ and some regular $\tilde{b} \in \tilde{A}$. But this follows from [GW, Lemma 5.1]. Thus $\tilde{A}$ is a right order in $\tilde{Q}$ and so by [GW, Theorem 5.10] $\tilde{A}$ is a semiprime, Goldie ring.

Step 5 ( $H$ cocommutative). We verify that $H$ is strongly semiprime with [C, Theorem 2]. Also by [C, Theorem 1] there is a finite extension field $L$ of $K$ such that $\tilde{H}=H \otimes_{K} L=L P^{*} \# L G$ which have been described in Step 3. Now we let $\tilde{H}$ act on $\tilde{A}$ as follows

$$
(h \otimes l) \cdot\left(a \otimes l^{\prime}\right)=h \cdot a \otimes l l^{\prime}
$$

for $h \in H, \quad l, l^{\prime} \in L$, and $a \in A$. See [BC].

Let $E$ be an essential right ideal of $A$. Since $E$ contains a regular element, $\tilde{E}=E \otimes_{K} L$ is an essential right ideal of $\tilde{A}$. By Step 3, $\tilde{E}$ contains an $\tilde{H}$-stable, essential right ideal $T^{\prime}$ of $\tilde{A}$. Let $\left\{u_{1}, \ldots, u_{m}\right\}$ be a $K$-basis of $L$. Let

$$
E_{i}=\left\{a \in E \mid \text { for some } t^{\prime} \in T^{\prime} \text { with } t^{\prime}=\sum_{j=1}^{m} a_{j} \otimes u_{j}, a_{i}=a\right\},
$$

for each $1 \leq i \leq m$. In other words, $E_{i}$ is the right ideal of coefficients of $u_{i}$ in $T^{\prime}$. For each $i, E_{i}$ is an $H$-stable right ideal of $A$ by the definition of the action of $\tilde{H}$ on $\tilde{A}$. We let

$$
\bar{E}=\sum_{i=1}^{m} E_{i}
$$

Now $\bar{E} \subseteq E$ and $\bar{E}$ is an $H$-stable right ideal of $A$. We show $\bar{E}$ is essential by noting

$$
T^{\prime} \subseteq \bar{E} \otimes L \subseteq E \otimes L=\tilde{E} .
$$

Thus $\bar{E} \otimes L$ is essential in $\tilde{A}$. Let $0 \neq R$ be a right ideal of $A$. Then $(R \otimes$ $L) \cap(\bar{E} \otimes L) \neq 0$. By comparing coefficients of $u_{i}$ 's, we see $\bar{E} \cap R \neq 0$. Thus $E$ is an essential $H$-stable right ideal of $A$ contained in $E$.

Of course, group algebras are cocommutative Hopf algebras and the above result applies. However, the assumption that the field is perfect is not needed for group algebras. We do not know if the assumption that $K$ is perfect is really needed for cocommutative actions.

Corollary 2.6 (Group Actions). Let $A$ be a $K$-algebra where $K$ is a field. Let $G$ be a finite group that acts on $A$, as $K$-automorphisms. Assume the order of $G$ is nonzero in $K$ and $K$ is a splitting field for $G$. Let $A$ be semiprime 
and right Goldie; then $\operatorname{CS}(A, K G)=\operatorname{CS}(Q(A), K G)$ where $Q(A)$ is the right classical quotient ring of $A$.

Proof. Finite group actions are $K G$ actions, where $K G$ is the group algebra. If $K G$ is semisimple, then it is strongly semiprime [P2, Theorem 4.4]. Let $A$ be a $K G$-module algebra. If $E$ is an essential right ideal of $A$, let $\bar{E}=\bigcap_{g \in G} E^{g}$. Then $\bar{E}$ is a $K G$ stable essential right ideal of $A$ contained in $E$.

In our next lemma, we make the same assumptions about $A$ and $H$, as we did in Theorem 1. Recall an algebra is called $H$-prime if the product of nonzero $H$-stable ideals is nonzero.

Lemma 2.7. $A$ is $H$-prime if and only if $Q(A)$ is $H$-prime.

Proof. Assume $A$ is $H$-prime; and let $\hat{J}, \hat{L}$ be $H$-stable ideals of $Q(A)$ with $\hat{J} \hat{L}=0$. Let $J=\hat{J} \cap A$ and $L=\hat{L} \cap A$. Then $J$ and $L$ are $H$-stable ideals of $A$ with $J L=0$. Thus $\hat{J}=0$ or $\hat{L}=0$.

Conversely, if $Q(A)$ is $H$-prime with $J$ and $L$ are $H$-stable ideals of $A$ with $J L=0$, then $L T^{-1}$ is an $H$-stable ideal of $Q(A)$. Now we note the left annihilator of $L T^{-1}$ is $H$-stable [OPQ, Lemma 1.4]. Since $J$ annihilates $L T^{-1}$, we have $J=0$ or $L=0$.

Our next result combines the main result of [OPQ, Theorem 1.6] and Theorem 5.

Theorem 2.8. Assume $H$ is a finite-dimensional, semisimple Hopf algebra over a splitting field $K$. Let $H$ be either commutative or cocommutative. If $H$ is cocommutative, we assume $K$ is a perfect field. Let $A$ be a semiprime $H$ module algebra with identity. Let $A$ be a right Goldie ring with quotient ring $Q$. Then $A \# H$ is prime if and only if $Q \# H$ is simple.

Proof. Assume $A \# H$ is prime. Thus by the main result of [OPQ], $\mathrm{CS}(A, H)=$ $\operatorname{Irr}(H)$ and $A$ is $H$-prime. Now we use the results of this paper to show $\operatorname{CS}(Q, H)=\operatorname{Irr}(H)$ and $Q$ is $H$-prime. So the main result of [OPQ] shows $Q \# H$ is prime. Since $Q \# H$ is Artinian, we have that it is simple. The converse follows by reversing the steps of this argument.

The group action case. For finite group actions, we have the following. Let $A$ be a $K$-algebra where $K$ is a field. Let $G$ be a finite group that acts on $A$, as $K$-automorphisms. Assume the order of $G$ is nonzero in $K$ and $K$ is a splitting field for $G$. Then if $A$ is semiprime and right Goldie, $A \# H$ is prime if and only if $Q \# H$ is simple, where $Q$ is the right classical quotient ring of $A$ and $H=K G$.

Theorem 2.9. Assume $H$ is a strongly semiprime, finite-dimensional Hopf $K$ algebra, where $K$ is a splitting field. Let $Q$ be semisimple Artinian, and let $Q$ be an $H$-module algebra. Then the hereditary subalgebras of $Q$ are of the form $f Q f$, where $f^{2}=f \in Q^{H}$. Furthermore, if $Q$ is $H$-simple, then $\operatorname{CS}(Q, H)=$ $\operatorname{Irr}(H)$ if and only if $B / B^{H}$ is $H^{*}$-Galois for every hereditary subalgebra $B$ of $Q$.

Proof. We show $Q^{H}$ is semisimple, Artinian, which is equivalent to showing there are no proper essential right ideals. Since $Q^{H}$ is Goldie, by Theorem 5, 
we need to show that regular elements of $Q^{H}$ are invertible. Let $t$ be regular in $Q^{H}$. Then there is an $s \in Q$ such that $t s=s t=1$. Now $1=\operatorname{Tr}(s) t=t \operatorname{Tr}(s)$. Thus $t$ is invertible in $Q^{H}$.

It is well known that ideals in semisimple, Artinian rings are generated by central idempotents. Let $J \neq 0$ be an ideal of $Q^{H}$, say $J=Q^{H} f$ for a central idempotent $f \in Q^{H}$. Note $B=J Q J=f Q f$, so each hereditary subalgebra has an identity. The $H^{*}$-Galois condition follows from [OPQ, Theorem 4.9] and [M1, Theorem 2.1].

We close this section with some remarks about finite group actions, and we show that the Connes spectrum for such actions on commutative domains is completely determined by the kernel of the action and vice versa. We let $G$ be a finite group and $K$ be a splitting field for $G$, with the order of $G$ invertible in $K$. Let $A$ be a $K$-algebra, and assume $A$ is prime. We say $G$ acts on $A$ if there is a group homomorphism

$$
\tau: G \rightarrow \operatorname{Aut}_{K}(A),
$$

where $\operatorname{Aut}_{K}(A)$ denotes the $K$-automorphisms of $A$. We let $W$ be the kernel of $\tau$. Now let $\operatorname{CS}(A, G)=\left\{\pi_{1} \cdots \pi_{t}\right\}$, and let $N=\bigcap_{i=1}^{t} \operatorname{ker}\left(\pi_{i}\right)$, where $\operatorname{ker}\left(\pi_{i}\right)$ denotes the kernel of $\pi_{i}$.

Lemma 2.10. $W \subseteq N$.

Proof. Let $\pi \in \operatorname{CS}(A, G)$ and $X \in A_{\pi}^{l}$. Then for $w \in W, X=w(X)=$ $\pi(w) X$. So $I-\pi(w)=0$ by [OP2, Theorem 5.7]. Thus $w \in N$.

Now $G / W$ acts on $A$ and by [OP2, Lemma 3.6], we have

$$
\operatorname{CS}(A, G / W)=\operatorname{CS}(A, G) \cap \operatorname{Irr}(G / W) .
$$

Also [OQ, Theorem 7], shows $\operatorname{CS}(A, G)=\operatorname{Irr}(G / N)$. For the definition of $X$-outer see [P2].

Theorem 2.11. Assume $G / W$ acts as $X$-outer automorphisms on $A$. Then $W=$ $N$.

Proof. We apply [OP2, Theorem 4.6] and conclude that $\operatorname{CS}(A, G / W)=$ $\operatorname{Irr}(G / W)$. Thus $\operatorname{Irr}(G / W)=\operatorname{Irr}(G / N)$ and hence $W=N$.

The domain case. Now we assume, in addition to the above, that $A$ is a commutative domain with quotient field $Q$. Then $G / W$ is an $X$-outer action of $Q$. So we have that the Connes spectrum is $\operatorname{Irr}(G / W)$. We note the spectrum is full if and only if the action is faithful and the spectrum is trivial if and only if the action is trivial.

\section{The CONNES SPECTRUM OF THE $H$-SYMMETRIC QUOTIENT RING}

In papers [Co], [M2], and [MS], an $H$-symmetric quotient ring $Q_{H}(A)$ is, constructed and the action $H$ on $A$ is extended (when the antipode is bijective) to $Q_{H}(A)$ to make it an $H$-module algebra.

We begin by describing some of the results of papers [M2] and [MS]. We let $H$ be a commutative or cocommutative finite-dimensional semisimple, Hopf algebra over a splitting field $K$. We note the antipode is bijective [M3, Corollary 
5.2.11]. Also $H$ is strongly semiprime, if $H$ is semisimple; see [P2, Theorem 4.8] and [MS, Corollary 7.13]. Let $A$ be an $H$-semiprime $H$-module algebra, and let $\mathscr{F}_{H}$ denote the set of two-sided $H$-stable ideals of $A$ that are regular in $A$. The left $H$-Martindale quotient ring of $A$ is

$$
Q_{H}^{l}(A)=\underset{I \in \mathscr{F}_{H}}{\underset{\lim }{\longrightarrow}} \operatorname{Hom}\left(I_{A}, A_{A}\right) .
$$

In particular, for all $q \in Q_{H}^{l}(A)$ there is $I \in \mathscr{F}_{H}$ such that $q I \subseteq A$. Now let

$$
Q_{H}(A)=\left\{q \in Q_{H}^{l}(A) \mid \text { there is } I \in \mathscr{F}_{H} \text { with } I q \subseteq A\right\} .
$$

This ring is called the $H$-symmetric quotient ring; see [Co], [M2], and [MS].

We next extend the action of $H$ to $Q_{H}(A)$. Let $q: I \rightarrow A$ for $I \in \mathscr{F}_{H}$ and $h \in H$. Then

$$
h \cdot q(a)=\sum_{(h)} h_{1} \cdot\left[q\left(S\left(h_{2}\right) \cdot a\right)\right]
$$

for all $a \in I$. Papers [Co, Proposition 6] and [M2, Corollary 3.5] shows that $Q_{H}(A)$ is an $H$-module algebra.

The following lemma is known, but we include it in the precise form we need.

Lemma 3.1. Assume $A^{H}$ is prime.

(1) Then $A$ is $H$-prime.

(2) Let $J$ be a nonzero ideal of $A^{H}$. Then $J$ is regular in $A$.

(3) Let $z \in Q_{H}(A)$, and assume $J z=0$ or $z J=0$ for some nonzero ideal $J$ of $A^{H}$. Then $z=0$.

Proof. Let $I, J$ be $H$-stable ideals of $A$ such that $I J=0$. $\operatorname{Then} \operatorname{Tr}(I) \operatorname{Tr}(J)=$ 0 and thus $I=0$ or $J=0$ by [OPQ, Proposition 4.3].

(2) The left or right annihilator of $J$ in $A$ is $H$-stable. Since $A^{H}$ is prime, the trace of the left or right annihilator must be zero.

(3) Assume $z J=0$ and find $I \in \mathscr{F}_{H}$ with $I z \subseteq A$. Then $(I z) J=0$ and by the first part of this lemma $I z=0$. Hence $z=0$. If $J z=0$ the proof is similar.

Lemma 3.2. Let $H$ be a finite-dimensional, semisimple commutative or cocommutative Hopf algebra over a splitting field $K$; and let $A$ be an $H$-module algebra with 1. Assume that $A$ is $H$-semiprime. If $0 \neq J$ is an ideal of $A^{H}$ and $B=J A J, \pi \in \operatorname{Irr}(H)$, and $C=\pi(H)$, then the following are equivalent:

(1) $B_{\pi}^{l} B_{\pi}^{r} \mathrm{reg} B_{\pi}^{m}$.

(2) $B_{\pi}^{l} B_{\pi}^{r} \mathrm{reg}(B \otimes C)$.

(3) $1 . \operatorname{ann}_{B_{\pi}^{m}}\left(B_{\pi}^{l}\right)=0=$ r.ann $B_{\pi}^{m}\left(B_{\pi}^{r}\right)$.

(4) $\operatorname{l.ann}_{(B \otimes C)}\left(B_{\pi}^{l}\right)=0=\mathrm{r} \cdot \operatorname{ann}_{(B \otimes C)}\left(B_{\pi}^{r}\right)$.

Proof. In the cocommutative case this is [OP2, Theorem 5.7]. So we assume $H$ is commutative. We begin by noting $C=K$, so $B \otimes C=B$. It is also easily seen that $B_{\pi}^{m}=B^{H}$. We show $B_{\pi}^{l}$ and $B_{\pi}^{r}$ are $H$-stable. Let $b \in B_{\pi}^{l} \subseteq B$ and $z \in H$. Then for any $h \in H, \sum_{(h)} \pi\left(h_{2}\right)\left(h_{1} \cdot(z \cdot b)\right)=z \cdot\left(\sum_{(h)} \pi\left(h_{2}\right)\left(h_{1} \cdot b\right)\right)=$ $z \cdot(\varepsilon(h) b)=\varepsilon(h)(z \cdot b)$. Thus $B_{\pi}^{l}$ is $H$-stable, and the proof for $B_{\pi}^{r}$ is similar.

(1) implies (2). Clearly $B_{\pi}^{l} B_{\pi}^{r}$ is $H$-stable, so $I=1 \cdot \operatorname{ann}_{B}\left(B_{\pi}^{l} B_{\pi}^{r}\right)$ is $H$-stable. But $I \cap B^{H}=0$, thus $I=0$, by [OPQ, Propostion 4.3(ii)]. The proof for the right annihilator is similar. 
(2) implies (3). Clear.

(3) implies (4). Now $I=1 . \mathrm{ann}_{B}\left(B_{\pi}^{l}\right)$ is $H$-stable [OPQ, Lemma 1.4]. But $B_{\pi}^{m}=B^{H}$.

(4) implies (1). Let $z \in B_{\pi}^{m}$ such that $z B_{\pi}^{l} B_{\pi}^{r}=0$; then $J=B_{\pi}^{r} z B_{\pi}^{l}$ is an ideal of $B^{H}$, by [OPQ, Lemma 5.1]. We note $J^{2}=0$ and $B^{H}$ is semiprime, thus $J=0$. Now (1) follows.

Theorem 3.3. Let $H$ be a commutative or cocommutative, finite-dimensional, semisimple Hopf algebra over a splitting field $K$. Let $A$ be $H$-module algebra such that $A^{H}$ is prime. Then $C S(A, H)=C S\left(Q_{H}(A), H\right)$.

Proof. We will let $Q$ denote $Q_{H}(A)$, and we note $Q$ is $H$-semiprime. This follows because $A$ is $H$-semiprime and a $0 \neq H$-stable ideal of $Q$ intersects $A$ in an $H$-stable, nonzero ideal of $A$.

Let $\pi \notin \operatorname{CS}(Q, H)$. Then by Lemma 2.3 , there is $\hat{J} \neq 0$ an ideal of $Q^{H}$ and $\hat{B}=\hat{J} Q \hat{J}$ such that $\operatorname{ann}_{\hat{B}_{\pi}^{m}}\left(\hat{B}_{\pi}^{l} \hat{B}_{\pi}^{r}\right) \neq 0$. Let $0 \neq z \in \operatorname{ann}_{\hat{B}_{\pi}^{m}}\left(\hat{B}_{\pi}^{l} \hat{B}_{\pi}^{r}\right)$.

Let $J=\hat{J} \cap A$, and note $J$ is a nonzero ideal of $A^{H}$. We let $B=J A J$. So $B \subseteq \hat{B}$. Thus

$$
\left(B_{\pi}^{l} B_{\pi}^{r}\right) z \subseteq\left(\hat{B}_{\pi}^{l} \hat{B}_{\pi}^{r}\right) z=0 .
$$

Now there is an $I \in \mathscr{F}_{H}$ such that $0 \neq z I \subseteq M_{n}(A)$. Let $w \in Q$ be a nonzero element of the $d_{\pi} \times d_{\pi}$ matrix $z$. Now by Lemma 3.1, $J w I^{H} J \neq 0$ and we note $0 \neq J z I^{H} J \subseteq M_{n}(B)$. Since $I^{H} J$ is an ideal of $A^{H}$ and $z \in \hat{B}_{\pi}^{m}$, it follows that $J z I^{H} J \subseteq B_{\pi}^{m}$. But

$$
\left(B_{\pi}^{l} B_{\pi}^{r}\right)\left(J z I^{H} J\right) \subseteq\left(B_{\pi}^{l} B_{\pi}^{r} z\right)\left(I^{H} J\right)=0 .
$$

Thus $\pi \notin \operatorname{CS}(A, H)$.

To show the other inclusion we need the hypothesis that $H$ is cocommutative or commutative. If $\pi \notin \operatorname{CS}(A, H)$ there is a nonzero ideal $J$ of $A^{H}$ such that the left annihilator of $B_{\pi}^{l}$ or the right annihilator of $B_{\pi}^{r}$ in $M_{n}(B)$ is nonzero, where $B=J A J$ and $n$ is the degree of $\pi$, by Lemma 3.2. Let $0 \neq z \in M_{n}(B)$ and $z B_{\pi}^{l}=0$.

Let $\hat{J}=Q^{H} J Q^{H}$ and $\hat{B}=\hat{J} Q \hat{J}$. Applying Lemma 3.1 to the entries of the nonzero matrix $z$, we have $z J \neq 0$. Let $x \in \hat{B}_{\pi}^{l}$ and take $C \in \mathscr{F}$ such that $x C \subseteq M_{n}(A)$. Let $I=C^{H} J$, which is nonzero because $A^{H}$ is prime. We observe

$$
J x I \subseteq J\left(x C^{H}\right) J \subseteq M_{n}(B) .
$$

Also $J x I \neq 0$ by Lemma 3.1, and one checks $J x I \subseteq B_{\pi}^{l}$. Thus $z J x I=0$ and so $z J x=0$. Thus $z J \hat{B}_{\pi}^{l}=0$. So if $\pi \in \operatorname{CS}(Q, H)$, then $z J=0$, by Lemma 3.2. Thus $z=0$ by Lemma 3.1, but $z \neq 0$. The situation for right annihilators is handled in the same way. Thus $\pi \notin \operatorname{CS}(Q, H)$.

We note that algebraically closed fields are splitting fields. So the next result yields Theorem B of the introduction.

Corollary 3.4. Let $H$ be a finite-dimensional, semisimple, commutative or cocommutative Hopf algebra over a splitting field $K$. Let $A$ be an $H$-module algebra that is $H$-semiprime. Then $A \# H$ is prime if and only if $Q_{H}(A) \# H$ is prime. 
Proof. Assume $A \# H$ is prime; then $A^{H}$ is prime and $Q_{H}(A)$ is $H$-prime. Thus by [OPQ, Theorem 1.6] and Theorem 3.3, $\operatorname{CS}(Q, H)=\operatorname{Irr}(H)$. This means $Q_{H}(A) \# H$ is prime.

Let $Q=Q_{H}(A)$, and assume $Q \# H$ is prime. We begin by showing $A^{H}$ is prime. Let $I_{1}$ and $I_{2}$ be ideals of $A^{H}$ with $I_{2} I_{1}=0$. If $I_{1} \neq 0, \quad I_{2} \neq 0$, then $I_{1} Q^{H} I_{2} \neq 0$, because $Q^{H}$ is prime. Let $q \in Q^{H}$ and $i \in I_{2}$ with $I_{1} q i \neq 0$. Choose $C \in \mathscr{F}_{H}$ with $q i C \subseteq A$. So $q i C^{H} \subseteq A^{H}$, and we will show $I_{1} q i C^{H} \neq 0$.

To do this we show the annihilator of $C^{H}$ in $Q$ is zero. Let $s \in Q$ with $s C^{H}=0$. Take $J \in \mathscr{F}$ such that $J s \subseteq A$; so $J s C^{H}=0$. Let

$$
L=\left\{a \in A \mid a C^{H}=0\right\} .
$$

Note $L$ is an $H$-stable left ideal of $A$. If $L \neq 0$, then $L^{H} \neq 0$, by [OPQ, Proposition 4.3]. But $C$ is regular in $A$ and so $L^{H} C \neq 0$. Now

$$
\operatorname{Tr}\left(L^{H} C\right)=L^{H} \operatorname{Tr}(C) \subseteq L C^{H}=0 .
$$

Thus $L^{H} C=0$. Thus $L=0$ and hence $J s=0$, which means $s=0$. Thus we have shown $I_{1} q i C^{H} \neq 0$.

But $q i C^{H} I_{1} \subseteq q i I_{1} \subseteq I_{2} I_{1}=0$. Thus $I_{1} q i C^{H}$ is a nonzero nilpotent left ideal of $A^{H}$. This contradicts the fact that $A^{H}$ is semiprime. Thus $A^{H}$ is prime. Hence by Lemma 3.1 $A$ is $H$-prime. By [OPQ, Theorem 1.6] and Theorem 3.3 $\operatorname{CS}(A, H)=\operatorname{Irr}(H)$. Hence $A \# H$ is prime.

We conclude by applying our results to pointed cocommutative Hopf algebra actions. In this situation, $Q_{H}(A)=Q(A)$, where $Q(A)$ is the symmetric quotient ring [MS, Example 3.5]. We have extended [P2, Theorem 15.9].

Corollary 3.5. Let $H$ be a finite-dimensional semisimple, pointed cocommutative Hopf algebra over a splitting field $K$. Let $A$ be an $H$-module algebra that is $H$-semiprime. Then $A \# H$ is prime if and only if $Q(A) \# H$ is prime.

\section{REFERENCES}

[BC] J. Bergen and M. Cohen, Actions of commutative Hopf algebras, Bull. London Math. Soc. 18 (1986), 159-164.

[C] W. Chin, Crossed products of semisimple cocommutative Hopf algebras, Proc. Amer. Math. Soc. 116 (1992), 321-327.

[Co] M. Cohen, Smash products, inner actions and quotient rings, Pacific Math. J. 125 (1986), 45-66.

[CR] M. Cohen, and L. Rowen, Group graded rings, Comm. Algebra 11 (1983), 1253-1270.

[GW] K. R. Goodearl and R. B. Warfield, Jr., An introduction to noncommutative Noetherian rings, Cambridge Univ. Press, Cambridge, 1989.

[M1] S. Montgomery, Hopf Galois extensions, Contemp. Math. 124 (1992), 129-140.

[M2] _ Bininvertible actions of Hopf algebras, Israel J. Math. 83 (1993), 45-71.

[M3] _ Hopf algebras and their actions, CBMS Regional Conf. Ser. in Math., no. 82, Amer. Math. Soc., Providence, RI, 1993.

[MS] S. Montgomery and H. J. Schneider, Hopf crossed products, rings of quotients, and prime ideals (to appear).

[OP1] J. Osterburg and D. S. Passman, What makes a skew group ring prime?, Azumaya Algebras, Actions, and Modules (D. E. Haile and J. Osterburg, eds.), Contemp. Math., vol. 124, Amer. Math. Soc., Providence, RI, 1992, pp. 165-177. 
[OP2] _ _ Computing the Connes spectrum of a Hopf algebra, Israel J. Math. 80 (1992), $225-253$.

[OPQ] J. Osterburg, D. S. Passman, and D. Quinn, A Connes spectrum for Hopf algebras, Contemp. Math. 130 (1992), 311-334.

[OQ] J. Osterburg and D. Quinn, Cocommutative Hopf algebra actions and the Connes spectrum, J. Algebra 165 (1994), 465-475.

[P1] D. S. Passman, Algebraic structure of group rings, Academic Press, Boston, MA, 1989.

[P2] _ Infinite crossed products, Academic Press, Boston, MA, 1989.

[Ph] N. C. Phillips, Equivariant $K$-theory and freeness of group actions on $C^{\star}$-algebras, Lecture Notes in Math., vol. 1274, Springer-Verlag, New York, 1987.

Department of Mathematical Sciences, University of Cincinnati, Cincinnati, Ohio 45221-0025

E-mail address: james.osterburg@uc.edu 